\title{
Trapping and survival probability in two dimensions
}

\author{
Lazaros K. Gallos and Panos Argyrakis \\ Department of Physics, University of Thessaloniki, GR-54006 Thessaloniki, Greece \\ Klaus W. Kehr* \\ Institut für Festkörperforschung, Forschungszentrum Jülich GmbH, D-52425 Jülich, Germany
}

(Received 28 January 2000; published 19 January 2001)

\begin{abstract}
We investigate the survival probability $\Phi(n, c)$ of particles performing a random walk on a two-dimensional lattice that contains static traps, which are randomly distributed with a concentration $c$, as a function of the number of steps $n . \Phi(n, c)$ is analyzed in terms of a scaling ansatz, which allows us to locate quantitatively the crossover between the Rosenstock approximation (valid only at early times) and the asymptotic DonskerVaradhan behavior (valid only at long times). While the existence of the crossover has been postulated before, its exact location has not been known. Our scaling hypothesis is based on the mean value of the quantity $S_{n}$, the number of sites visited in an $n$-step walk. We make use of the idea of self-interacting random walks, and a "slithering" snake algorithm, available in the literature, and we are thus able to obtain accurate survival probability data indirectly by Monte Carlo simulation techniques. The crossover can now be determined by our method, and it is found to depend on a combination of $c$ and $n$. It occurs at small $\Phi(n, c)$ values, which is typically the case for large values of $n$.
\end{abstract}

DOI: 10.1103/PhysRevE.63.021104

PACS number(s): 05.40.Fb, 72.10.-d

The trapping problem continues to be one of the most puzzling problems of transport in disordered systems [1-16]. The problem is the precise characterization of the survival probability of a particle that performs a random walk in the presence of randomly distributed static traps. This problem acquires its complications from the interplay of two different random events: the random distribution of traps leads to the formation of large trap-free regions [7]. Additionally, there is a finite set of random walks within these regions. We consider here the lattice version of the trapping problem. Common sense suggests an exponential decay of the survival probability with the number of steps (time), except perhaps in one dimension. The simplest approximate treatment of the problem (Rosenstock [8]) yields an exponential dependence on time (number of steps $n$ ) in dimensions $d \geqslant 3$, and exponential dependence on $\sqrt{n}$ in $d=1$. There exists a rigorous treatment of the problem by Donsker and Varadhan [1]; their result is an exponential decay of the survival probability with an argument proportional to $n^{d /(d+2)}$. Hence, simple exponential decay would appear only in infinite dimensions, but in all finite dimensions the result would be different from the Rosenstock approximation. The Donsker-Varadhan result is an asymptotic one, valid only in the limit $n \rightarrow \infty$. No direct simulations of the trapping problem have ever shown the asymptotic Donsker-Varadhan behavior [5], whereas at small concentrations of traps and step numbers the Rosenstock approximation seems to be applicable. Hence the important question arises as to the nature of the crossover from the Rosenstock approximation to the rigorous asymptotic result. This is specifically the goal of the present article.

As will be detailed below, a satisfactory description of the crossover between the small- $n$ behavior and the case $n \rightarrow \infty$ exists in $d=1$ [6]. Also, in $d=3$ a satisfactory picture has

\footnotetext{
*Deceased.
}

emerged [14]. In this paper we will study the notoriously difficult case of $d=2$ by making a scaling analysis. We emphasize that the two-dimensional case is important for diffusion processes on surfaces, which are of great current interest.

We consider hypercubic lattices in arbitrary dimensions $d$ (linear, square, simple cubic, etc.) where a finite fraction of sites, designated as trap sites, is randomly distributed on the lattice. The concentration of the trap sites shall be $c$. The trap sites are considered as static, i.e., we have quenched disorder. A particle is placed randomly on the lattice and it performs a regular random walk. When the particle reaches a trap site it gets absorbed by it irreversibly, i.e. it is annihilated. The survival probability $\Phi(n, c)$ is the probability that a particle has not been trapped (i.e., it survives) after a random walk of $n$ steps, on a lattice containing a concentration $c$ of such traps.

The first step in deriving the survival probability $\Phi(n, c)$ can be made by a very simple consideration, which has been known for a long time. It utilizes the number of distinct sites visited at least once in an $n$-step random walk, $S_{n}$. For a concentration $c$ of traps $(0<c<1)$, each lattice site has a probability $1-c$ of not being a trap. If a particle has survived in a random walk of $n$ steps, then none of the $S_{n}$ sites visited can be a trap site. This event has the probability

$$
P_{n}=(1-c)^{S_{n}}
$$

Thus, the survival probability is exactly

$$
\Phi(n, c)=\left\langle(1-c)^{S_{n}}\right\rangle=\left\langle e^{-\lambda S_{n}}\right\rangle,
$$

where $\lambda=-\ln (1-c)$. The remaining average in Eq. (2) is over different realizations of the random walks of the particle; the average over the trap distributions has already been performed. 
Clearly, to solve this problem it suffices to have the complete distribution $p_{n}(S)$ of the values of $S_{n}$ in different walks. Thus Eq. (2) can be written in the form

$$
\Phi(n, c)=\sum_{S} p_{n}(S)(1-c)^{S} .
$$

This distribution is known only in one dimension [3], but not in two or three dimensions. A first approximation, proposed by Rosenstock [8], consists in replacing the quantity $S_{n}$ in Eq. (2) by its mean value $\left\langle S_{n}\right\rangle$,

$$
\Phi(n, c)=\left\langle(1-c)^{S_{n}}\right\rangle \simeq(1-c)^{\left\langle S_{n}\right\rangle} .
$$

The average values $\left\langle S_{n}\right\rangle$ are known accurately for all dimensionalities, and for any $n[17,21]$. However, it turns out that Eq. (4) is not in satisfactory agreement with precise simulation data, as it applies only to low trap concentrations and early times.

The Rosenstock approximation can be improved by using the cumulant generating function [9]

$$
K(\lambda, n)=\sum_{j=1}^{\infty}(-1)^{j} \frac{\lambda^{j}}{j !} k_{j}(n),
$$

where $k_{j}(n)$ are the so-called cumulants. The survival probability can then be written as

$$
\Phi(n, c)=\exp [K(\lambda, n)] .
$$

In practice, the upper index has to be truncated from $\infty$ to a value in the range $j=2-4$. This formula yields better results, but it requires the moments $\left\langle S_{n}^{j}\right\rangle$, which also are not known analytically.

Actually, Donsker and Varadhan [1] have proved an exact result for $\Phi(n, c)$, which is valid for all dimensionalities, but its validity is restricted to the long-time limit. The survival probability is rigorously given as

$$
\lim _{n \rightarrow \infty} \Phi(n, c)=\exp \left(-K_{d} \lambda^{2 /(2+d)} n^{d /(d+2)}\right),
$$

where $d$ is the dimensionality and $K_{d}$ is a positive constant depending on the dimensionality and the structure of the lattice. Notice that Eq. (7) is a limiting expression for $n \rightarrow \infty$, and no information is given on when this limit is reached.

The asymptotic behavior of the survival probability can be derived from qualitative, Flory-type, arguments that were developed by Grassberger and Procaccia [7]. We will not reproduce the argument here, except for the remark that the behavior results from the combination of the distribution of trap-free regions and of random walks that are restricted to these regions. Also, Nieuwenhuizen [16] studied analytically the behavior of $\Phi$ in three-dimensional lattices. He was able to estimate crossover times for small trap concentrations, which verified the numerical results of Ref. [14]. The crossover times reported in [16] are very large as derived by a renormalization theory analysis.

The rigorous result of Donsker and Varadhan is in apparent contradiction to the Rosenstock approximation for the survival probability. For instance, in one dimension Donsker and Varadhan predict an argument in the exponent $\sim n^{1 / 3}$ while the Rosenstock approximation gives $\sim n^{1 / 2}$; in $d=3$ the arguments of the exponent behave as $\sim n^{3 / 5}$ and $\sim n$, respectively. How can the two results be tied together? This problem can be treated more or less completely in $d=1$, where sufficiently complete information on the distribution $p_{n}(S)$ of the distinct sites visited is available [3].

Using the asymptotic expression for $p_{n}(S)$, Anlauf [6] derived an asymptotic expansion of the survival probability, which is in excellent agreement with the simulations. We reproduce the form given in Ref. [19] (in this reference an extension with regard to a correlated walk was made; here we restrict the formula to an uncorrelated walk),

$$
\begin{aligned}
\Phi(n, c)= & \frac{c^{2}}{(1-c) \lambda^{2}} 8\left(\frac{2}{3}\right)^{1 / 2}\left(\frac{x}{\pi}\right)^{3 / 2} \\
& \times \exp \left[-\frac{3 x}{2}+\frac{a_{1}}{x}+\frac{a_{2}}{x^{2}}+\cdots\right] .
\end{aligned}
$$

The scaling variable is $x=(\pi \lambda)^{2 / 3} n^{1 / 3}$ and the coefficient $a_{1}$ is given by

$$
a_{1}=\frac{\pi^{2} \lambda^{2}}{12}
$$

The next coefficient can be found in [19].

In $d=3$ the crossover between the Rosenstock approximation and the asymptotic limit described by the DonskerVaradhan result was studied in terms of a scaling analysis by Anlauf [14]. The details of this analysis will be described below, where it will be extended to the more complicated case of $d=2$.

The two-dimensional case is difficult for a theoretical description, because the random walk represents the marginal case of recurrent behavior. This is reflected in the presence of logarithmic terms in the mean number $\left\langle S_{n}\right\rangle$ of distinct sites visited $[17,21]$, which lead to difficulties in the scaling analysis.

In order to study the crossover between the Rosenstock approximation and the asymptotic Donsker-Varadhan behavior, we perform a scaling analysis of $\Phi(n, c)$, in a similar way to that done by Anlauf in $d=3$. The starting point is the notion of a self-interacting random walk (SIW), which was first introduced by Stanley et al. [15]. The method was further elaborated by Meirovitch [20]. In a SIW every random walk that visits $S$ sites has a total statistical weight $e^{-\lambda S}$, where $\lambda$ is a parameter representing a form of dimensionless energy. Thus, we can construct the partition function as

$$
Z=\sum_{\text {all walks }} e^{-\lambda S} .
$$

The mean value $\langle S\rangle_{\text {SIW }}$ in the case of a SIW is given by

$$
\langle S\rangle_{\mathrm{SIW}}=\frac{1}{Z} \sum_{\text {all walks }} S e^{-\lambda S}=-\frac{\partial \ln Z}{\partial \lambda} .
$$


The SIW takes place on a trap-free lattice. However, if we interpret $\lambda$ as $-\ln (1-c)$ we can correlate the SIW with the random walk on a lattice with traps of concentration $c$. We then observe that the survival probability can be written as

$$
\Phi(n, c)=\frac{1}{(2 d)^{n}} Z,
$$

and thus

$$
-\ln \Phi(n, c)=n \ln 2 d-\ln Z .
$$

If we integrate Eq. (11) with respect to $\lambda$ we get

$$
\begin{aligned}
\int_{0}^{\lambda}\langle S\rangle_{\mathrm{SIW}} d \lambda^{\prime} & =-\int_{0}^{\lambda} d \ln Z \\
& =-\left.\ln Z\right|_{\lambda^{\prime}=\lambda}+\left.\ln Z\right|_{\lambda^{\prime}=0} \\
& =-\ln Z+\ln \sum_{\text {all walks }} e^{-0 S}=-\ln Z+\ln (2 d)^{n} .
\end{aligned}
$$

The final part of this equation is exactly the same as the right part of Eq. (13), so by substituting in (13) we have our final result,

$$
-\ln \Phi(n, c)=\int_{0}^{\lambda}\langle S\rangle_{\mathrm{SIW}} d \lambda^{\prime}
$$

where $\lambda=-\ln (1-c)$. This formula relates the survival probability to the mean number of sites visited during a SIW. This is a very important step, since we need to compute only an average value for different values of $\lambda$ and not a distribution in complete detail, as needed in direct Monte Carlo simulations of trapping.

In order to overcome attrition problems with the numerical calculation of $\langle S\rangle_{\text {SIw }}$ we implement a "slithering snake" algorithm [18]. First, we fix the number of steps $n$, and we create a normal $n$-step random walk starting from a given point ("tail") and resulting in the "head" site. This chain (or snake), comprised of $n$ bonds connecting $n+1$ sites not necessarily different, starts moving and transforming its shape. The head picks a random neighboring position and decides whether it will move there. If the move is accepted the rest of the chain moves along the original chain, leaving the old tail site vacant. The decision to accept the new configuration is based on $\Delta S$, which measures the difference in sites occupied by the chain before and after the move of the head. The possible values of $\Delta S$ are $-1,0,1$. A move is always accepted when $\Delta S<1$. If $\Delta S=1$, which means that the chain will grow longer, the move is accepted with a probability $p=e^{-\lambda \Delta S}=e^{-\lambda}=1-c$, where $c$ is the fixed trap concentration for this walk. When the move is not accepted it has been shown $[14,18]$ that the chain should simply change its direction of moving by interchanging the labels of head and tail. We repeat the same procedure and monitor the number of sites occupied by the chain for uncorrelated configurations. Thus, after acquiring many values of $S$, we can de-

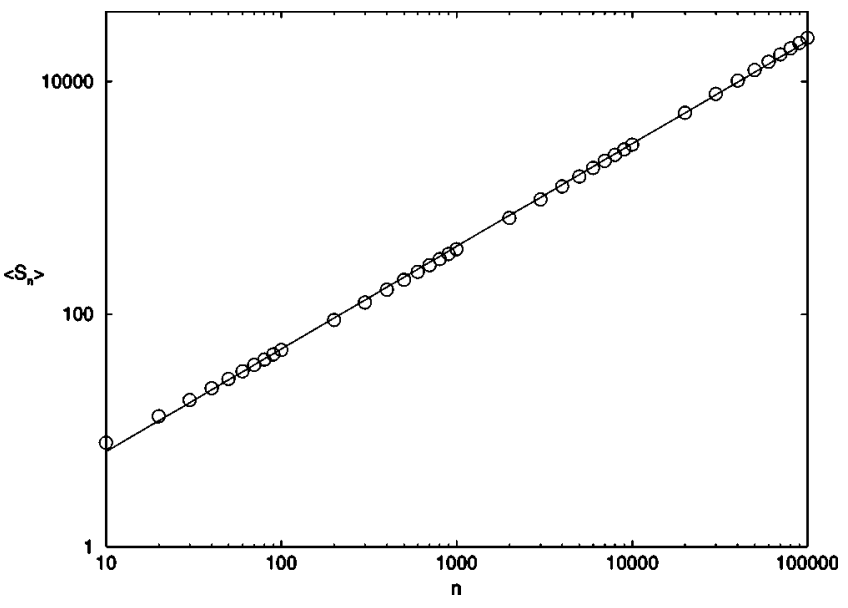

FIG. 1. Fitting of the $\left\langle S_{n}\right\rangle$ data derived from the Henyey and Seshadri paper [21] (symbols) to a power law (solid line).

termine $\langle S\rangle_{\text {SIW }}$ for a given value of $c$. All we have to do now in order to compute $\Phi(n, c)$ is to repeat the entire algorithm for different values of $c$ and integrate the resulting curve according to Eq. (15).

A scaling analysis can be performed as follows. We know that in the small- $n$ limit the Rosenstock approximation is quite accurate, while in the large- $n$ limit the DonskerVaradhan result is valid. Thus, a scaling form of $\Phi(n, c)$ should tend to the following limit behaviors:

$$
-\ln \Phi(n, c) \sim\left\{\begin{array}{l}
\lambda\left\langle S_{n}\right\rangle, \quad \text { small } n \\
k_{d} \lambda^{2 /(d+2)} n^{d /(d+2)}, \quad n \rightarrow \infty .
\end{array}\right.
$$

For $d=1$ or $d=3\left\langle S_{n}\right\rangle$ follows a power law and it is relatively easy to perform a scaling ansatz of the form

$$
-\ln \Phi(n, c)=n^{\alpha} f\left(\lambda n^{\beta}\right),
$$

but for $d=2$ the logarithmic denominator in the formula for $\left\langle S_{n}\right\rangle$ makes such an approach impossible. However, one can try to fit $\left\langle S_{n}\right\rangle$ in $d=2$ in the early-time regime also with a power law, since we are not really interested in the exact form of $\left\langle S_{n}\right\rangle$, but rather in its general behavior in this regime. For this fit, we use the formula for $\left\langle S_{n}\right\rangle$ given by Henyey and Seshadri [21], which gives a very accurate representation of $\left\langle S_{n}\right\rangle$ in $d=2$ over an extended region of $n$. The result of the fit is $\left\langle S_{n}\right\rangle \sim 0.72 n^{0.9}$ and the quality of the fit is satisfactory, as shown in Fig. 1, at least for the purpose of this work. If we assume this form of $\left\langle S_{n}\right\rangle$ to be applicable for the scaling analysis, we find $\alpha=0.10$ and $\beta=0.80$. This results in

$$
-\frac{\ln \Phi}{n^{0.1}}=f\left(\lambda n^{0.8}\right)=f(x) \sim\left\{\begin{array}{l}
A x, \quad x \rightarrow 0 \\
k_{d} x^{1 / 2}, \quad x \rightarrow \infty
\end{array}\right.
$$

where $x=\lambda n^{0.8}$ is the scaling variable. By combining Eqs. (11), (13), and (18) we get

$$
\langle S\rangle_{\mathrm{SIW}}=-\frac{\partial \ln Z}{\partial \lambda}=n^{0.9} f^{\prime}\left(\lambda n^{0.8}\right),
$$




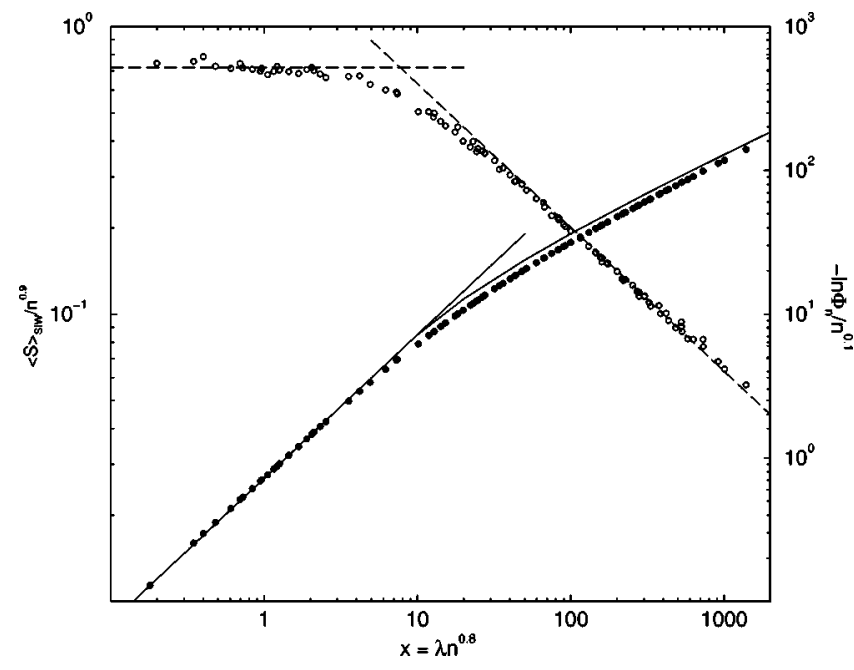

FIG. 2. Double logarithmic plot of $\langle S\rangle_{\text {SIW }} / n^{0.9}$ (left ordinate axis) and $-\ln \Phi(n, c) / n^{0.1}$ (right ordinate axis) as a function of the scaling variable $x$. The open circles are the results of simulations using the slithering snake algorithm for different trap concentrations ranging from $c=0.005$ to $c=0.9$. The two dashed lines are the ones predicted by Eq. (20), while the solid lines are those of Eq. (22). The filled circles are the result of numerically integrating the open circles.

where

$$
\frac{\langle S\rangle_{\mathrm{SIW}}}{n^{0.9}}=f^{\prime}(x) \sim\left\{\begin{array}{l}
A, \quad x \rightarrow 0 \\
\frac{k_{d}}{2} x^{-1 / 2}, \quad x \rightarrow \infty .
\end{array}\right.
$$

This formula means that if we plot $\langle S\rangle_{\mathrm{SIW}} / n^{0.9}$, as derived by the simulations, as a function of $\lambda n^{0.8}$, we expect to observe initially a constant value and, after a crossover regime, a power law with slope -0.5 . This will also be a strong indication that the asymptotic limit has been reached.

In Fig. 2 we present this plot for different numbers of steps and different trap concentrations. We can see that indeed the behavior is as expected, thus verifying the proposed assumptions.

We can transform Eq. (15) according to our scaling variables,

$$
-\ln \Phi(n, c)=n^{0.1} \int_{0}^{x\langle S\rangle_{\mathrm{SIW}}} \frac{n^{0.9}}{n^{2}}
$$

The crossover point $x_{c}$ can be found by the intersection of the two lines in Fig. 2. The result is $x_{c} \simeq 8.76$ and the integral can be separated into two parts $\left(x \leqslant x_{c}\right.$ and $\left.x>x_{c}\right)$. We use Eq. (20) to get

$$
\frac{-\ln \Phi(n, c)}{n^{0.1}}=\left\{\begin{array}{l}
A x, \quad x \leqslant x_{c} \\
A x_{c}+k_{d}\left(x^{1 / 2}-x_{c}^{1 / 2}\right), \quad x>x_{c} .
\end{array}\right.
$$

This two-part function is also represented in Fig. 2, where the solid circles are the result of the numerical integration of the open circles in the same figure. In Fig. 3 we present the numerically calculated effective exponent of $x$ in Eq. (18),

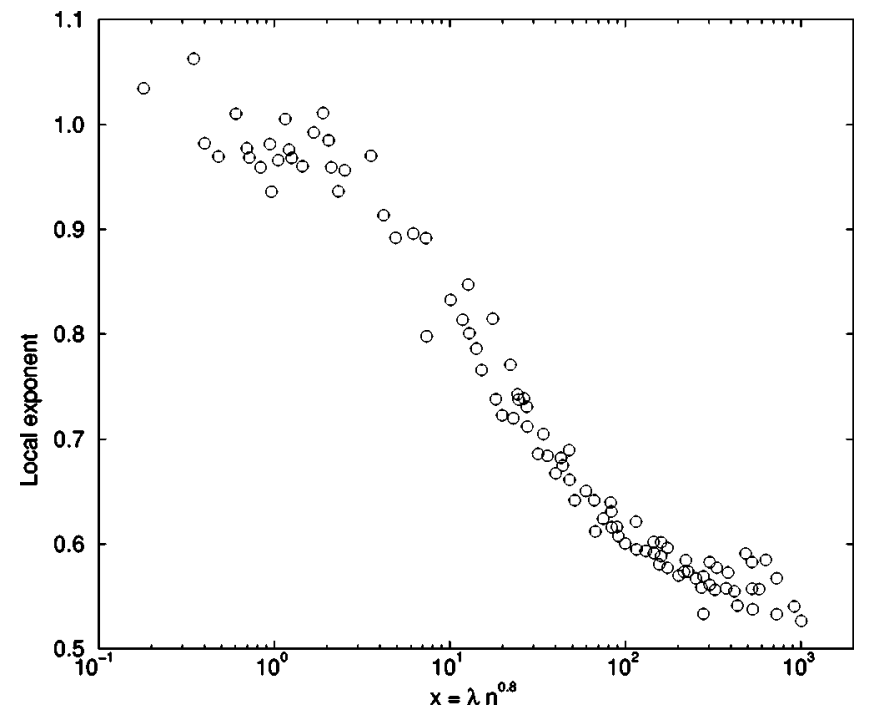

FIG. 3. Local exponent of $x$ in Eq. (18), as a function of $x$ $=\lambda n^{0.8}$, derived numerically from the points in Fig. 2 .

which is the slope of our data in Fig. 2. We observe that the slope initially has a value around 1 , while asymptotically it tends to the value 0.5 . Although this asymptotic value has not been exactly achieved, the slope at $x=1000$ is very close to 0.5 . Therefore, we can claim that the Donsker-Varadhan limit is reached for values $x_{D V} \simeq 1000$ or higher.

Figure 3 should be compared to Fig. 2(a) of Ref. [5]. In this figure, we can see that the local slope initially reduces toward the value 0.5. However, it is soon masked by the exponential term, finally yielding a slope of 1 , although in larger systems smaller values of the local exponent are reached. Our method, though, is not really influenced by finite size problems and thus the local slope exponent in Fig. 3 is monotonically decreasing, allowing us to directly locate the onset of the Donsker-Varadhan regime. It is also interesting to note that when $c=0.5$ the number of steps corresponding to the value $x_{D V}=1000$ that we derived in our work is roughly 9000, which is in quite good agreement with the value acquired if we extrapolate the data of Fig. 2(a) in Ref. [5].

Summarizing, in this paper we have investigated the trapping problem in $d=2$. It is well known, and we also discussed in detail, that the theoretical treatment for the case of dimensionality $d=2$ is more demanding than in other dimensions, because this is the borderline dimension for recurrent random walks. For instance, the mean number of distinct sites visited by a random walk of $n$ steps, $\left\langle S_{n}\right\rangle$, contains logarithmic terms.

To obtain insight into the crossover between the Rosenstock approximation, which is valid for short times and small trap concentrations, and the asymptotic Donsker-Varadhan behavior, we made a scaling analysis of the behavior of the survival probability in the complete time-concentration domain. The method of analysis was based on the methods introduced by Anlauf for the case $d=3$ [14]. Such an analysis is particularly difficult in $d=2$ because of the presence of logarithmic terms in the quantity $\left\langle S_{n}\right\rangle$, which enters the Rosenstock approximation. We circumvented the problem of 
the logarithmic terms by fitting $\left\langle S_{n}\right\rangle$ to a power law over a rather large interval of $n$. Although this procedure is partially heuristic, a scaling form of $\Phi(n, c)$ was obtained. This scaling form could then be used to determine the crossover between the Rosenstock approximation and the asymptotic Donsker-Varadhan expression. As expected, the crossover occurs at values of step numbers and concentrations where the survival probability is already very small. We have succeeeded in exploring the survival probability in $d=2$ into the asymptotic Donsker-Varadhan regime by relating it to a selfinteracting walk. This method proves quite powerful, since it does not suffer from finite size effects, and it is valid in a very wide time regime and a wide range of trap concentrations, all in the most general way. We believe that the direct determination of the survival probability in $d=2$, either by simulations or by experiments, is extremely difficult, if not impossible [5]. Alternatively, the direct analytical determination of the complete distribution of $S_{n}$ seems also a very difficult task. Only indirect methods such as the present one can succeed in this complex problem.

We thank Professor J. Anlauf for several discussions. We also thank the University of Paderborn (Germany) Computer Center for a computing grant used to carry out part of the calculations.
[1] M.D. Donsker and S.R.S. Varadhan, Commun. Pure Appl. Math. 28, 525 (1975); 32, 721 (1979).

[2] F. den Hollander and G.H. Weiss, in Contemporary Problems in Statistical Physics, edited by G.H. Weiss (SIAM, Philadelphia, 1994).

[3] G.H. Weiss and R.J. Rubin, Adv. Chem. Phys. 52, 363 (1983).

[4] G.H. Weiss, Aspects and Applications of the Random Walk (North-Holland, Amsterdam, 1994).

[5] A. Bunde, S. Havlin, J. Klafter, G. Graff, and A. Shehter, Phys. Rev. Lett. 78, 3338 (1997).

[6] J.K. Anlauf, Phys. Rev. Lett. 52, 1845 (1984).

[7] P. Grassberger and I. Procaccia, J. Chem. Phys. 77, 6281 (1982).

[8] H.B. Rosenstock, J. Math. Phys. 11, 487 (1970).

[9] G. Zumofen and A. Blumen, Chem. Phys. Lett. 88, 63 (1982).

[10] R.F. Kayser and J.B. Hubbard, Phys. Rev. Lett. 51, 79 (1983).
[11] D.C. Torney, J. Stat. Phys. 44, 49 (1986).

[12] T.C. Lubensky, Phys. Rev. A 30, 2657 (1984).

[13] S. Havlin, M. Dishon, J.E. Kiefer, and G.H. Weiss, Phys. Rev. Lett. 53, 407 (1984).

[14] J.K. Anlauf, doctoral dissertation, University of Cologne, 1988.

[15] H.E. Stanley, K. Kang, S. Redner, and R.L. Blumberg, Phys. Rev. Lett. 51, 1223 (1983).

[16] Th.M. Nieuwenhuizen, Phys. Rev. Lett. 62, 357 (1989).

[17] E.W. Montroll and G.H. Weiss, J. Math. Phys. 6, 167 (1965).

[18] F.T. Wall and F. Mandel, J. Chem. Phys. 11, 4592 (1975).

[19] K.W. Kehr, J.K. Anlauf, and R. Czech, in Transport and Relaxation in Random Materials, edited by J. Klafter, R.J. Rubin, and M.F. Shlesinger (World Scientific, Singapore, 1986).

[20] H. Meirovitch, Phys. Rev. A 32, 3699 (1985).

[21] F.S. Henyey and V. Seshadri, J. Chem. Phys. 76, 5530 (1992). 